\title{
Efficient Adsorptive Performance of Medical Stone Decorated by Carbon Dots
}

\author{
Baolong Zhao*, Leilei Hu*, Hengjia Kang* and Zhihong Zheng*(**)† \\ *School of Environmental and Municipal Engineering, North China University of Water Resources and Electric Power, \\ Zhengzhou 450011, China \\ **Henan Vocational College of Water Conservancy and Environment, Zhengzhou 450008, China \\ †Corresponding author: Zhihong Zheng; zzh@ncwu.edu.cn
}

\section{Nat. Env. \& Poll. Tech. Website: www.neptjournal.com \\ Received: 20-08-2019 \\ Revised: 21-09-2019 \\ Accepted: 11-12-2019}

\section{Key Words:}

Adsorptive performance

Carbon dots

Medical stone

Organic pollutants

\begin{abstract}
Carbon dots could significantly change the property of a normal material and have received wide attention in the recent decade. In this research, glucose as a carbon source, carbon dots decorated medical stone (CD-MS) was successfully synthesized for efficient adsorptive removal of organic pollutants. Pyrolytic temperature and glucose concentration for the adsorbent preparation were proved to have a significant impact on the adsorptive performance. The optimal pyrolytic temperature and glucose concentration were found to be $300^{\circ} \mathrm{C}$ and $0.5 \mathrm{M}$, yielding the optimized adsorbent $0.5 \mathrm{CD}$ MS-300 superior to other carbon dots decorated MS. Surface morphology analysis demonstrated that the carbon dots were successfully immobilized on the surface of MS while the atomic ratio of C increased from $2.6 \%$ of the raw MS to $11.25 \%$ of the $0.5 \mathrm{CD}-\mathrm{MS}-300$. Three organic pollutants including p-nitrophenol, orange II and methylene blue with different charge properties were employed to explore the adsorptive performance of the 0.5CD-MS-300. The results indicated that the surface of 0.5CDMS-300 was negatively charged while carbon dots had significantly improved the adsorption capability of the raw MS. As such, the resulting adsorbent 0.5CD-MS-300 can be considered as a powerful adsorbent for the removal of some organic contaminants from wastewater.
\end{abstract}

\section{INTRODUCTION}

With the development of industry and agriculture, a large number of organic contaminants, such as dyes, endocrine-disruptors, and pharmaceutical and personal care products, have come into the natural environment along with the discharge of effluents (Westerhoff et al. 2005, Crini 2006). These organic pollutants existing in wastewater can cause a severe hazard to human and animal health due to the delivery of the food chain. To date, there are many treatment technologies, including coagulation, sonocatalytic degradation, photocatalytic degradation and adsorption, to be applied to the removal of the organic pollutants (Polubesova et al. 2006, Li et al. 2014, Mahata et al. 2007, Zhang et al. 2006). Among these technologies, the adsorption process is considered as a promising and reliable method due to its ease of operation, low energy consumption, low cost and high efficiency (Qu 2008).

Medical stone (MS) is one kind of natural silicate mineral without toxicity. Its main mineral components include $\mathrm{Na}\left(\mathrm{AlSi}_{3} \mathrm{O}_{8}\right), \mathrm{Na}\left(\mathrm{AlO}_{2}\right)\left(\mathrm{SiO}_{2}\right)_{3}, \mathrm{~K}\left(\mathrm{AlSi}_{3} \mathrm{O}_{8}\right), \mathrm{K}\left(\mathrm{AlO}_{2}\right)$ $\left(\mathrm{SiO}_{2}\right)_{3}, \mathrm{Ca}\left(\mathrm{Al}_{2} \mathrm{Si}_{2} \mathrm{O}_{8}\right)$ and $\mathrm{Mg}\left(\mathrm{Al}_{2} \mathrm{Si}_{2} \mathrm{O}_{8}\right)$ (Gao et al. 2011). MS has a tetrahedron structure of $\left[\mathrm{SiO}_{4}\right]^{2}$ bonding to metal ions (such as $\mathrm{K}, \mathrm{Na}, \mathrm{Ca}, \mathrm{Mg}, \mathrm{Cu}$ and $\mathrm{Al}$, etc.), contributing to the formation of a large internal surface area (Gao et al. 2012). Therefore, a series of excellent performances for MS are expected, such as biological activity, the ability of dissolving and adsorbing and adjusting $\mathrm{pH}$ capacity. Meanwhile, it is widely used for food preservation, water purification and medical care. In recent years, MS had been studied as an adsorbent material, which achieved remarkable advances on the adsorption of heavy metals in wastewater (Gao et al. 2011, Zhou et al. 2015). Even though, to our best knowledge, there have been few reports about the adsorption removal of organic contaminants.

Carbon dots are novel carbon-based nanomaterial. They contain abundant oxygen-containing functional groups $(-\mathrm{OH}$, $-\mathrm{COOH}$ and $-\mathrm{C}=\mathrm{O}$ ) on the surfaces, with good solubilities and optical properties. As such, carbon dots are widely implemented for bioimaging, chemical sensors, and photovoltaic devices, etc. (Ding et al. 2014, Shen et al. 2012). In recent years, CDs have been considered an ideal candidate material for organic or inorganic pollutants removal due to their rich functional groups, ease of preparation and low toxicity (Hsu \& Chang 2012). However, some significant disadvantages of CDs based adsorbent focused on the dissolution of CDs as well as difficulties of separation and regeneration (Liu et al. 2017). Consequently, it is a necessity of combining CDs with other constituents to synthesize CDs-functionalized adsorbents. 
In this research, we prepared the carbon-dots decorated medical stone (CD-MS) for the efficient adsorption of organic pollutants. Glucose, with numerous hydroxy groups and formyl groups, was chosen as the precursor of CDs and can provide more active adsorption sites on the surfaces of the MS (Hsu \& Chang 2012). The effects of pyrolytic temperature and glucose concentration for the adsorbent preparation were emphatically studied. At the same time, p-nitrophenol (PNP), orange II (ORII) and methylene blue (MB) with different charge properties in a neutral aqueous solution were selected as target organic pollutants to investigate the adsorptive performance and adsorption capacity of the CD-MS.

\section{MATERIALS AND METHODS}

Chemicals: Orange II (ORII) (mass fraction > 95\%) was purchased from Beijing Chemical Reagents Company. Methylene blue (MB) (mass fraction $>98.5 \%$, chemical pure) was purchased from Tianjin Chemical Reagent Research Institute. P-nitrophenol (PNP) used was of analytical grade and provided by Tianjin Guangfu Fine Chemical Research Institute, China. Other chemicals were of analytical grade and used without further purification. Deionized (DI) water was used throughout the research. The molecular structures of ORII, MB and PNP are shown in Fig. 1.

Adsorbent preparation: The natural medical stone (MS) was obtained from the Hunan Province of China. It was shattered with a multi-function pulveriser and subsequently sieved to a particle size between those of 80 -mesh screens and 100-mesh screens. Firstly, $10 \mathrm{~g}$ of the powdered MS was immersed to $200 \mathrm{~mL}$ of $0.5 \mathrm{M}$ or $0.05 \mathrm{M}$ glucose solution and the mixture was shaken at a speed of $120 \mathrm{rpm}$ for $12 \mathrm{~h}$. Then, the resulting samples were rinsed with DI water for five times and oven-dried overnight at $80^{\circ} \mathrm{C}$. The prepared carbon dots decorated medical stone (CD-MS) are denoted as $0.5 \mathrm{CD}-\mathrm{MS}$ and $0.05 \mathrm{CD}-\mathrm{MS}$ in the following studies.

Desired amounts of MS, 0.5CD-MS and 0.05CD-MS were tightly placed in a ceramic crucible with a lid and pyrolyzed in a muffle furnace under oxygen-limited conditions. The pyrolysis temperature was raised to the desired
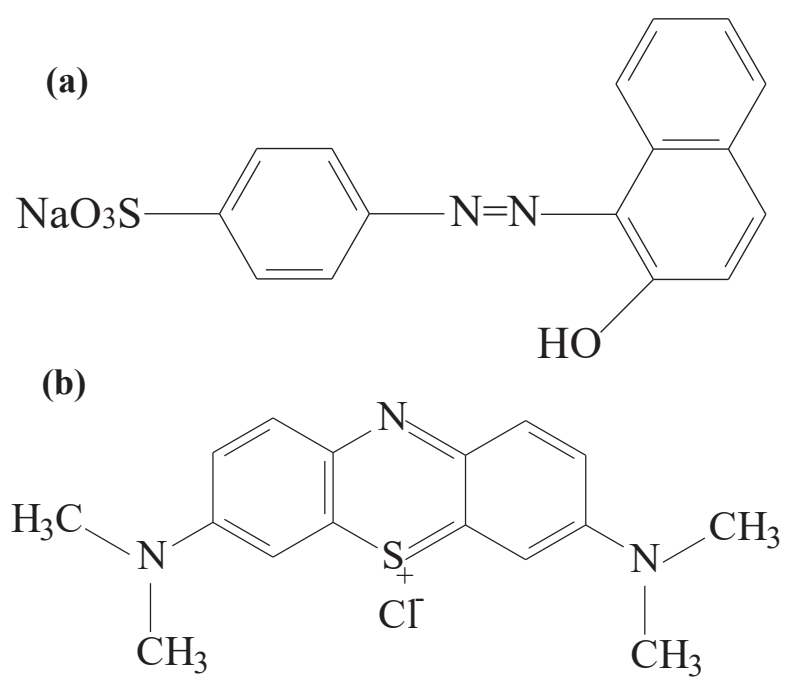

(c)

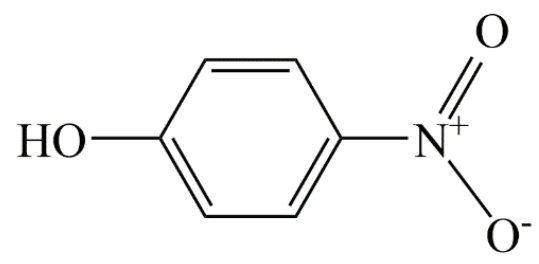

Fig. 1: The molecular structures of ORII (a), MB (b), and PNP (c). 
values of $200^{\circ} \mathrm{C}, 300^{\circ} \mathrm{C}$ and $400^{\circ} \mathrm{C}$ at a heating rate of $10^{\circ} \mathrm{C} \mathrm{min}^{-1}$ and held constant for $2 \mathrm{~h}$. After pyrolyzation, the samples were allowed to cool down to room temperature in the furnace. These pyrolyzed 0.05CD-MS were denoted as 0.05CD-MS-200, 0.05CD-MS-300 and 0.05CD-MS-400, respectively, while the pyrolyzed $0.5 \mathrm{CD}-\mathrm{MS}$ are denoted as 0.5CD-MS-200, 0.5CD-MS-300 and 0.5CD-MS-400, respectively. Similarly, the adsorbent MS-200, MS-300 and MS-400 were also prepared.

Batch adsorption studies: Batch experiments were conducted in a series of 250-mL conical flasks. In the flasks, 20 $\mathrm{mg}$ of the adsorbent was added to $100 \mathrm{~mL}$ solution with an initial PNP, ORII or MB concentration of $20 \mathrm{mg} / \mathrm{L}$. Then, the flasks were sealed and shaken at $298 \mathrm{~K}$ at a speed of $120 \mathrm{rpm}$ for $24 \mathrm{~h}$ to achieve equilibrium. Solution $\mathrm{pH}$ was maintained at neutral unless otherwise stated. To examine the effect of glucose concentration, $5 \mathrm{mg}$ adsorbent (0.5CDMS or 0.05CD-MS) was used to adsorb PNP with an initial concentration of $5 \mathrm{mg} / \mathrm{L}$.

Characterization: The morphology and superficial element compositions of MS and 0.5CD-MS-300 were characterized by a Hitachi S-4800 field scanning electron microscope (FESEM) coupled with an energy dispersive spectrometer (EDS).

Analysis methods: All the samples were collected and filtered by microporous membranes $(0.45 \mu \mathrm{m})$ before analysis. The concentration of PNP, ORII and MB was measured using UVmini-1240 spectrophotometer (Shimadzu, Japan) at the wavelength of maximum adsorption of $317 \mathrm{~nm}, 483$ $\mathrm{nm}$, and $664 \mathrm{~nm}$, respectively (Cheng et al. 2019, Mi et al.
2016). The adsorption capacity was calculated using the following equation:

$$
q_{e}=\left(\mathrm{C}_{0}-\mathrm{C}_{\mathrm{e}}\right) \mathrm{V} / \mathrm{M}
$$

Where, $q_{e}(\mathrm{mg} / \mathrm{g})$ is the adsorption capacity at equilibrium; $C_{0}$ and $C_{e}(\mathrm{mg} / \mathrm{L})$ are the initial and equilibrium concentrations of PNP, ORII, and MB, respectively; $V(\mathrm{~L})$ is the solution volume, and $M(\mathrm{~g})$ is the weight of adsorbents.

\section{RESULTS AND DISCUSSION}

\section{Effect of Pyrolytic Temperature and Glucose Concentration on PNP Adsorption}

Both the pyrolytic temperature and glucose concentration were important for adsorbent preparation. It was observed from Fig. 2 that the CD-MS pyrolyzed at $300^{\circ} \mathrm{C}$ had a comparatively higher adsorption capacity for PNP removal. The concentration of glucose for the adsorbent preparation was expected to have a significant impact on the uptake of contaminants as well. As illustrated, it was noted that the adsorption capacity of PNP by $0.5 \mathrm{CD}-\mathrm{MS}$ was higher than that by $0.05 \mathrm{CD}-\mathrm{MS}$ under the same pyrolytic temperature. Further, at the pyrolytic temperature of $300^{\circ} \mathrm{C}$, the adsorption capacities by the $0.5 \mathrm{CD}-\mathrm{MS}$ and $0.05 \mathrm{CD}-\mathrm{MS}$ were $4.16 \mathrm{mg} / \mathrm{g}$ and $1.88 \mathrm{mg} / \mathrm{g}$, respectively. This might be attributed to the increasing bonding sites on the CD-MS surfaces resulted from the increase of glucose concentration. Similar experimental results were also observed by Xue et al. (2013). As such, 0.5CD-MS-300 was used for the following experiments.

On the other hand, as the structure of pore canals and surface area of internal pores of CD-MS varied as a conse-

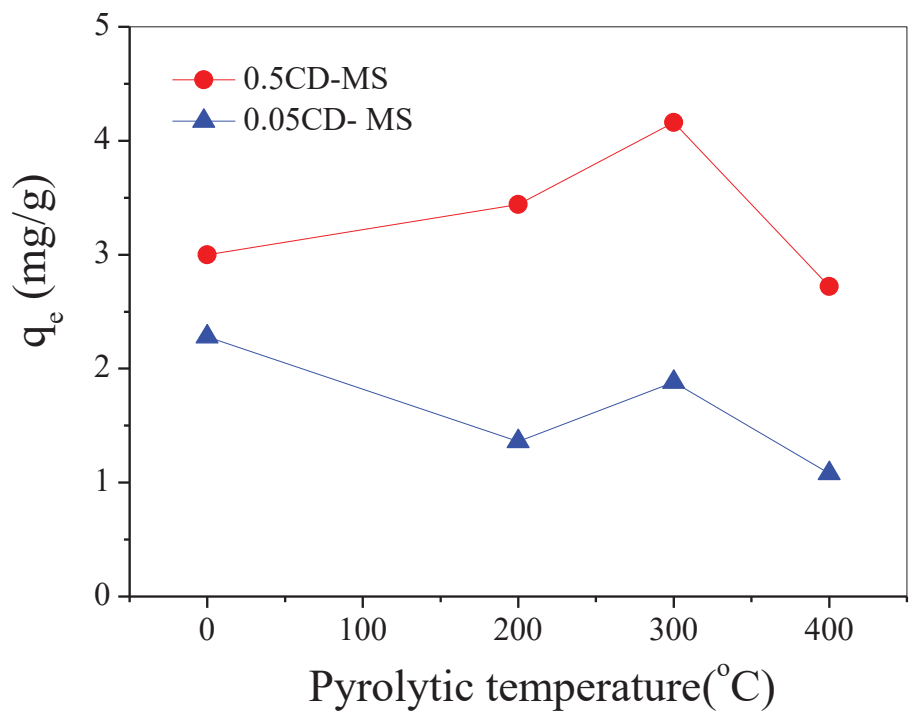

Fig. 2: Effect of glucose concentration and pyrolytic temperature on adsorption of PNP. PNP $5.0 \mathrm{mg} / \mathrm{L}$, neutral solution $\mathrm{pH}$. 
quence of the different pyrolytic temperatures, the adsorption capacity of PNP by the resulting adsorbents was expected to vary accordingly. The relationship between pyrolytic temperature and adsorption capacity of PNP is presented in Fig. 2. It shows that the adsorption capacity of PNP by $0.5 \mathrm{CD}$ MS-300 reached the maximum of $4.16 \mathrm{mg} / \mathrm{g}$, which might be attributed to the removal of crystal water in the pore canals and the increase of the surface area of internal pores as the pyrolytic temperatures rose. However, continuous increase of pyrolytic temperature was not helpful for the uptake of PNP and the adsorption capacity declined, which might be attributed to the collapse of pore canals.

\section{Characterization of the Carbon Dots Decorated Medical Stone}

As stated above, the 0.5CD-MS-300 demonstrated the highest adsorption capacity for PNP in comparison to other adsorbents. The surface morphology of the 0.5CD-MS-300 and the raw MS was characterized by SEM and presented herein. From Fig. 3a and c, it was observed that the raw MS presented a natural crystal structure, although it was difficult to find some pore canals from the MS surfaces. Differently, the surface structure of the 0.5CD-MS-300 became loose after pyrolysis, and some sponge pores were scattered onto the surfaces of the adsorbent, which might facilitate the adsorption of contaminants (Yu et al. 2013). This demonstrated pyrolysis treatment can change the surface structure of MS. From Fig. 3b and d, many fine particles aggregated on the surfaces of MS, which were deduced to be carbon dots immobilized on the surfaces of MS.

To further explore whether carbon dots had decorated on the surfaces of MS, EDS analysis was conducted. As illustrated in Fig. 4a and b, comparing to the atomic ratio of $\mathrm{C}$ increased from $2.6 \%$ of the raw MS to $11.25 \%$ of the $0.5 \mathrm{CD}$ MS-300, which was reasonably attributed to the introduction of CDs on the surfaces of MS. Accordingly, the atomic ratio of O decreased from $57.97 \%$ of the raw MS to $53.70 \%$ of the 0.5CD-MS-300. Pyrolysis under different temperatures and immobilization of carbon dots had significantly altered the surface properties of the raw MS.

\section{Effect of Adsorbent Dosage on PNP Adsorption}

The effect of adsorbent (0.5CD-MS-300) dosage from 100 $\mathrm{mg} / \mathrm{L}$ to $800 \mathrm{mg} / \mathrm{L}$ was investigated and presented in Fig. 5. The plot showed that uptake of PNP decreased gradually with an increase of adsorbent dosage. One reason was that the unsaturated adsorption sites increased with increasing adsorbent dosage at a fixed concentration and volume of
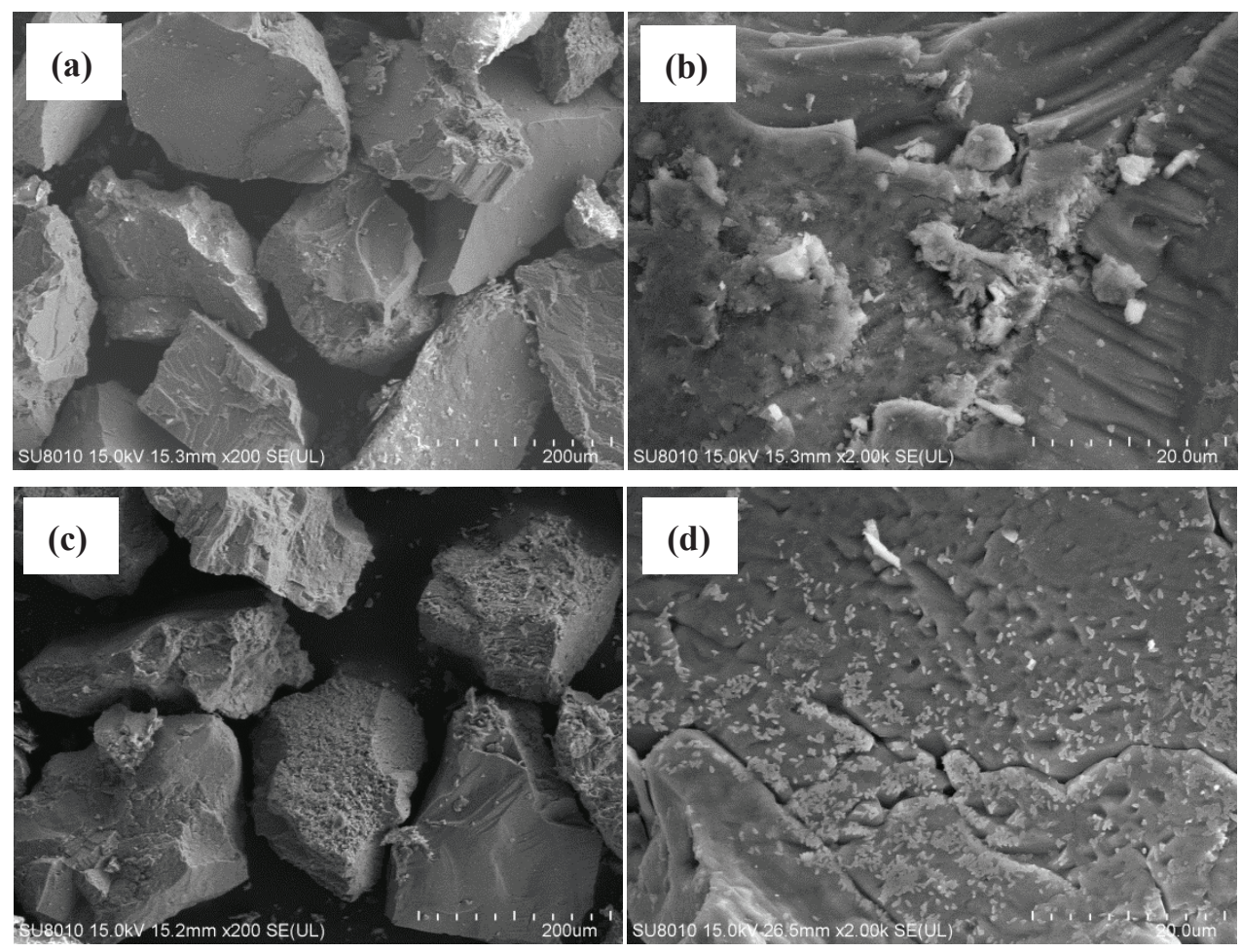

Fig. 3: SEM images of the raw MS (a, b) and the 0.5CD-MS-300 (c, d). 
PNP. The other was attributed to the decrease of surface area and the increase of diffusion path length of adsorbent due to the particle aggregation (Shirmardi et al. 2016, Kumar et al. 2010).

\section{Adsorptive Performance of Carbon Dots Decorated Medical Stone}

The surface charge property of an adsorbent always plays an important role during the adsorptive removal of organic contaminants. Three organic pollutants with different charge properties including PNP, ORII and MB were selected to explore the adsorptive performance of the 0.5CD-MS-300. The initial concentration of the three contaminants was fixed at $20 \mathrm{mg} / \mathrm{L}$. As illustrated in Fig. 6, it was noted that the adsorption capacity of PNP and MB by 0.5CD-MS-300 was much higher than that of ORII. The adsorption capacity of PNP, MB, and ORII was $27.87 \mathrm{mg} / \mathrm{g}, 15.37 \mathrm{mg} / \mathrm{g}$, and 0 $\mathrm{mg} / \mathrm{g}$, respectively. This indicated that the 0.5CD-MS-MS had a high adsorption selectivity for organic pollutants with different charge properties.

To our knowledge, PNP $\left(\mathrm{pK}_{\mathrm{a}}=7.15\right)$ in water solution exists in two species of molecule and anion, and molecular

(a)
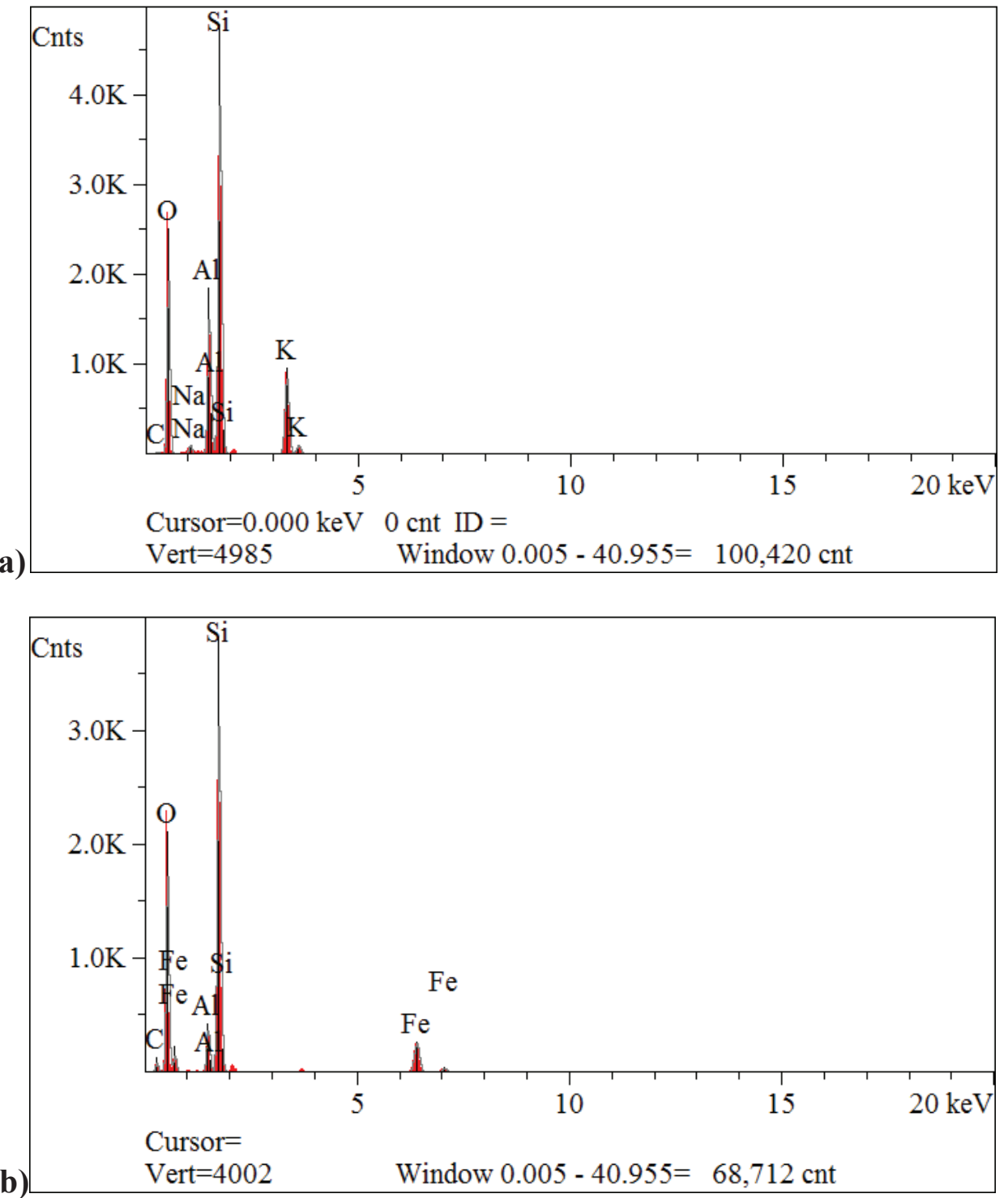

Fig. 4: EDS analysis results of the raw MS(a) and the 0.5CD-MS-300 (b). 


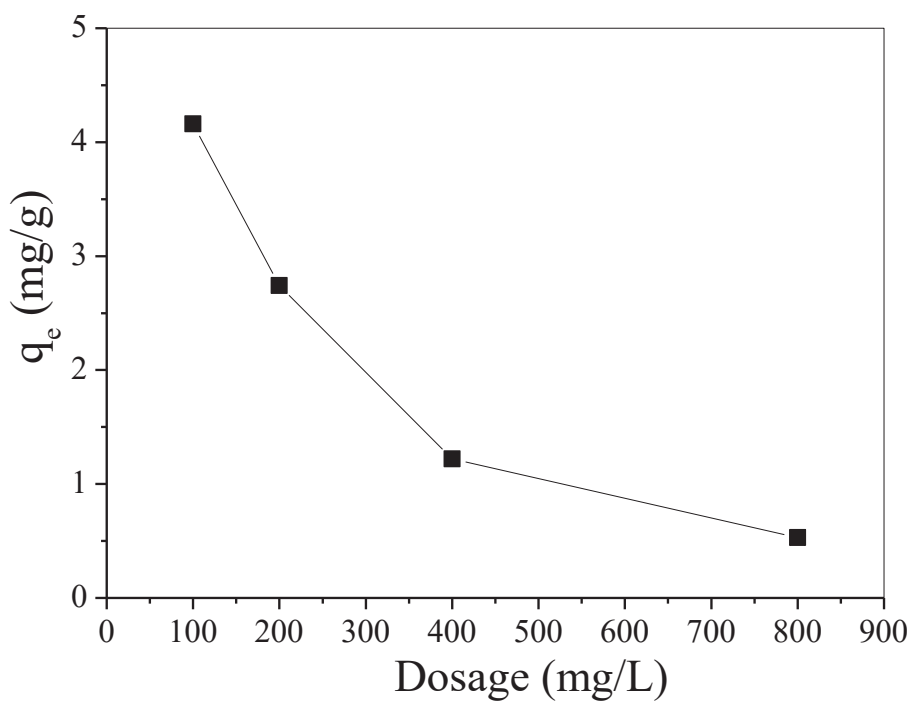

Fig. 5: Effect of adsorbent dosage on PNP adsorption. PNP $5.0 \mathrm{mg} / \mathrm{L}$, neutral solution $\mathrm{pH}$.
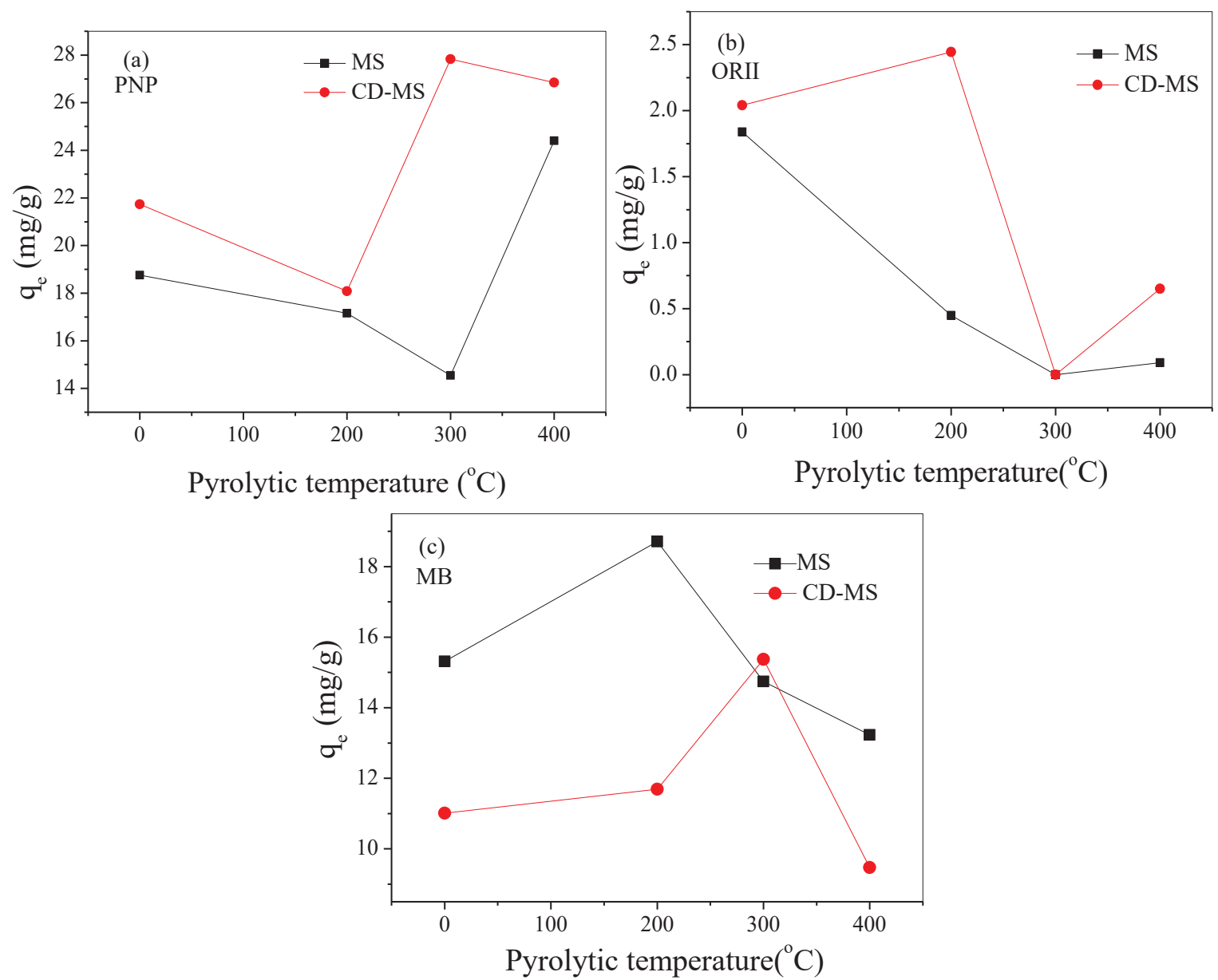

Fig. 6: Adsorption of PNP (a), ORII (b) and MB (c) onto the raw MS and the 0.5CD-MS-300.

Contaminant concentration $20.0 \mathrm{mg} / \mathrm{L}$, neutral solution $\mathrm{pH}$. 
PNP can convert to anions at $\mathrm{pH}>7.15$ due to dissociation (Sarkar et al. 2010). Meanwhile, MB is moderately alkaline in water and produces cation $\left(\mathrm{C}^{+}\right.$or $\left.\mathrm{CH}^{+}\right)$(Gobi et al. 2011). By contrast, ORII ( $\mathrm{pK}_{\mathrm{a}}=10.6$ and $\left.\mathrm{pK}_{\mathrm{a}}=1\right)$ has three different forms depending on the $\mathrm{pH}$ of the aqueous solution, noted $\mathrm{H}_{2} \mathrm{~L}$ which is doubly protonated and dominant at $\mathrm{pH}<1$, $\mathrm{HL}^{-}$which is mono protonated and dominant at $\mathrm{pH}$ 1-10.6, $\mathrm{L}^{2-}$ which is non-protonated and dominant at $\mathrm{pH}>10.6$ (Abramian \& El-Rassy 2009). Therefore, the three pollutants have different electrical characteristics in a neutral aqueous solution and the predominant species of PNP, MB and ORII are molecule, cation $\left(\mathrm{C}^{+}\right.$or $\left.\mathrm{CH}^{+}\right)$, and anion $\left(\mathrm{HL}^{-}\right)$, respectively. Accordingly, from the results mentioned above, it could be deduced that the surface of $0.5 \mathrm{CD}-\mathrm{MS}-300$ was negatively charged, which was beneficial to MB adsorption due to an electrostatic attraction. Meanwhile, the results indicated that electrostatic attraction/repulsion was an important adsorption mechanism for 0.5CD-MS-300, which also facilitated the high adsorption selectivity for these organic pollutants. However, an interesting phenomenon was that the adsorption capacity of PNP is excellent. As mentioned, most of PNP exists as molecules in a neutral solution, which indicated that the effect of electrostatic force was not dominant. Therefore, considering the abundant $\mathrm{O}$-containing functional groups of carbon dots on the 0.5CD-MS-300, the higher adsorption capacity of PNP might be attributed to H-bond interaction between PNP molecules and the surface functional groups of the adsorbent, $\pi$ - $\pi$ interaction, and $\pi$-stacking forces (Zheng et al. 2017).

In addition, the 0.5CD-MS-300 demonstrated a higher adsorption capacity towards PNP and ORII than the raw MS in general, indicating that the introduction of carbon dots was beneficial to the adsorption capability for PNP and ORII. By contrast, the introduction of carbon dots declined the adsorption of MB. Therefore, the surface of the 0.5CDMS-300 was predominantly negatively charged.

\section{CONCLUSION}

Carbon dots decorated medical stone (CD-MS) was creatively prepared by immobilizing carbon dots on the surfaces of medical stone (MS). Compared to the raw MS, the 0.5CDMS-300 demonstrated an excellent adsorption capability for PNP. Bach experiments for the adsorption of the three pollutants including PNP, ORII and MB with different charge properties, indicated that the surface of $0.5 \mathrm{CD}-\mathrm{MS}-300$ was negatively charged. Furthermore, $0.5 \mathrm{CD}-\mathrm{MS}-300$ had a high adsorption selectivity for different organic pollutants, which was attributed to the electrostatic attraction/repulsion as one of the fundamental adsorption mechanisms. In a word, carbon dots had successfully decorated the raw medical stone and the resulting adsorbent $0.5 \mathrm{CD}-\mathrm{MS}-300$ can be considered as a powerful adsorbent for the removal of organic pollutants from wastewater.

\section{ACKNOWLEDGEMENTS}

The authors thank for the financial support from the Major Science and Technology Project in Henan Province of China, the integration and demonstration of sewage treatment technology for typical villages and towns of Henan Province. (161100310700).

\section{REFERENCES}

Abramian, L. and El-Rassy, H. 2009. Adsorption kinetics and thermodynamics of azo-dye Orange II onto highly porous titania aerogel. Chem. Eng. J., 150: 403-410.

Cheng, M., Jiang, J.H., Wang, J.J. and Fan, J. 2019. Highly salt resistant polymer supported ionic liquid adsorbent for ultrahigh capacity removal of p-nitrophenol from water. ACS Sustain. Chem. Eng., 7: $8195-8205$

Crini, G. 2006. Non-conventional low-cost adsorbents for dye removal: A review. Bioresour. Technol., 97: 1061-1085.

Ding, C.Q., Zhu, A.W. and Tian, Y. 2014. Functional surface engineering of $\mathrm{C}$-dots for fluorescent biosensing and in vivo bioimaging. Acc. Chem. Res., 47(1): 20-30.

Gao, G.H., Lei, Y.H., Dong, L.H., Liu, W.C., Wang, X.F., Chang, X.T., Liu, T., Yin, Y.S. and Ajayan, P.M. 2012. Synthesis of nanocomposites of silver nanoparticles with medical stone and carbon nanotubes for their antibacterial applications. Mater. Express, 2(2): 2185-5849.

Gao, T.P., Wang, W.B. and Wang, A.Q. 2011. A pH-sensitive composite hydrogel based on sodium alginate and medical stone: Synthesis, swelling, and heavy metal ions adsorption properties. Macromol. Res., 19(7): 739-748.

Gobi, K., Mashitah, M.D. and Vadivelu, V.M. 2011. Adsorptive removal of methylene blue using novel adsorbent from palm oil mill effluent waste activated sludge: Equilibrium, thermodynamics and kinetic studies. Chem. Eng. J., 171: 1246-1252.

Hsu, P.C. and Chang, H.T. 2012. Synthesis of high-quality carbon nanodots from hydrophilic compounds: Role of functional groups. Chem. Commun. (Camb), 48: 3984-3986.

Kumar, P.S., Ramalingam, S., Senthamarai, C., Niranjanaa, M., Vijayalakshmi, P. and Sivanesan, S. 2010. Adsorption of dye from aqueous solution by cashew nut shell: Studies on equilibrium isotherm, kinetics and thermodynamics of interactions. Desalination, 261: 52-60.

Li, G.T., Zhao, W.G., Wang, B.B., Gu, Q.Y. and Zhang, X.W. 2014. Synergetic degradation of Acid Orange 7 by fly ash under ultrasonic irradiation. Desalin. Water Treat., 57(5): 1-8.

Liu, F.B., Zhang, W.T., Chen, W.J., Wang, J., Yang, Q.F., Zhu, W.X. and Wang, J.L. 2017. One-pot synthesis of $\mathrm{NiFe}_{2} \mathrm{O}_{4}$ integrated with EDTA-derived carbon dots for enhanced removal of tetracycline. Chem. Eng. J., 310: 187-196.

Mahata, P., Aarthi, T., Madras, G. and Natarajan, S. 2007. Photocatalytic degradation of dyes and organics with nanosized $\mathrm{GdCoO}_{3}$. J. Phys. Chem. C, 111: 1665-1674.

Mi, X., Li, G.T., Zhu, W.Y. and Liu, L.L. 2016. Enhanced adsorption of Orange II using cationic surfactant modified biochar pyrolyzed from cornstalk. Journal of Chemistry, 2016, 1-7.

Polubesova, T., Zadaka, D., Groisman, L. and Nir, S. 2006. Water remediation by micelle-clay system: Case study for tetracycline and sulfonamide antibiotics. Water Res., 40: 2369-2374. 
Qu, J.H. 2008. Research progress of novel adsorption processes in water purification: A review. J. Environ. Sci., 20: 1-13.

Sarkar, B., Xi, Y.F., Megharaj, M., Krishnamurti, G.S. and Naidu, R. 2010. Synthesis and characterisation of novel organopalygorskites for removal of p-nitrophenol from aqueous solution: isothermal studies. J. Colloid Inter. Sci., 350(1): 295-304.

Shen, J.H., Zhu, Y.H., Yang, X.L. and Li, C.Z. 2012. Graphene quantum dots: Emergent nanolights for bioimaging, sensors, catalysis and photovoltaic devices. Chem. Commun. (Camb), 48: 3686-3699.

Shirmardi, M., Alavi, N., Lima, E.C., Takdastan, A., Mahvi, A.H. and Babaei, A.A. 2016. Removal of atrazine as an organic micro-pollutant from aqueous solutions: A comparative study. Process Saf. Environ., 103: 23-35.

Westerhoff, P., Yeomin, Y., Snyder, S. and Wert, E. 2005. Fate of endocrine-disruptor, pharmaceutical, and personal care product chemicals during simulated drinking water treatment processes. Environ. Sci. Technol., 39: 6649-6663.
Xue, G.H., Gao, M.L., Gu, Z., Luo Z.X. and Hu, Z.C. 2013. The removal of p-nitrophenol from aqueous solutions by adsorption using gemini surfactants modified montmorillonites. Chem. Eng. J., 218: 223-231.

Yu, Q.J., Cui, C.Y., Zhang, Q., Chen, J., Li, Y., Sun, J.P., Li, C.Y., Cui, Q.K., Yang, C.H. and Shan, H.H. 2013. Hierarchical ZSM-11 with intergrowth structures: Synthesis, characterization and catalytic properties. J. Energy Chem., 22: 761-768.

Zhang, X., Li, A.M., Jiang, Z.M. and Zhang, Q.X. 2006. Adsorption of dyes and phenol from water on resin adsorbents: Effect of adsorbate size and pore size distribution. J. Hazard Mater., 137: 1115-1122.

Zheng, H.S., Guo, W.Q., Li, S., Chen, Y.D., Wu, Q.L., Feng, X.C., Yin, R.L., Ho, S.H., Ren, N.Q. and Chang, J.S. 2017. Adsorption of p-nitrophenols (PNP) on microalgal biochar: Analysis of high adsorption capacity and mechanism. Bioresource Technol., 244: 1456-1464.

Zhou, W.X., Guan, D.G., Sun, Y., Sun, C.M., Xu, G.L., Chen, T., Yu, Z.S., $\mathrm{Xu}$, Y. and Yan, H. 2015. Removal of Nickel (II) ion from wastewater by modified maifanite. Mater. Sci. Forum, 814: 371-375. 